\title{
COMPARAÇÃO ENTRE MÉTODOS DE AMOSTRAGEM DO SOLO PARA RECOMENDAÇÃO DE CALAGEM E ADUBAÇÃO DO CAFEEIRO CONILON ${ }^{1}$
}

\author{
RONE B. DE OLIVEIRA ${ }^{2}$, JULIÃO S. DE S. LIMA ${ }^{3}$, ALEXANDRE C. XAVIER ${ }^{3}$, \\ RENATO R. PASSOS ${ }^{4}$, SAMUEL DE A. SILVA ${ }^{5}$, ALESSANDRA F. DA SILVA ${ }^{5}$
}

\begin{abstract}
RESUMO: O objetivo deste trabalho foi comparar a metodologia convencional de amostragem de solo com a análise espacial para a recomendação de calagem e adubação de nitrogênio, fósforo e potássio em solo cultivado com café conilon. O experimento foi realizado nas safras de 2004/2005 e 2005/2006, em área de 1,0 ha, com as amostras retiradas na profundidade de 0-0,20 m. No método convencional, coletaram-se 15 subamostras em caminhamento ziguezague, constituindo uma amostra composta, e, no método espacial, construiu-se uma malha amostral de 109 pontos georreferenciados. Com os resultados das análises, foi calculada a necessidade de calagem e adubação em função do teor do elemento no solo e da produtividade esperada das plantas. Os dados foram analisados por meio da estatística clássica (descritiva e exploratória) e pela análise espacial, utilizando técnicas de geoestatística (modelagem da estrutura de variabilidade espacial e realização de inferências) e geoprocessamento (álgebra de mapas). Com exceção da recomendação de fósforo em 2005 e potássio em 2006, todas as demais recomendações apresentaram dependência espacial. A análise dos dados pelo método espacial possibilitou identificar zonas de déficit ou excesso de calagem e de adubação na área, que não poderiam ser definidas com o método convencional de amostragem (ziguezague).
\end{abstract}

PALAVRAS-CHAVE: geoestatística, geoprocessamento, manejo do solo.

\section{COMPARISON BETWEEN SOIL SAMPLING METHODS FOR CONILON COFFEE LIMING AND FERTILIZATION RECOMMENDATION}

\begin{abstract}
This study aimed to evaluate the soil sampling conventional methodology with spatial analysis for liming and fertilization with nitrogen, phosphorus and potassium on soil cultivated with conilon coffee. The trial was carried out during the 2004/2005 - 2005/2006 harvests in a 1.0 ha area with samples collected at $0-0.20 \mathrm{~m}$ depth. Fifteen sub samples in zigzag were collected for the conventional method forming a compound sample; as for the spatial method, 109 georeferenced points formed a sample grid. After the analysis results, the liming and fertilization needs were calculated based on the function of the element content in the soil and on the plant expected yield. Data were analyzed by both the classical statistics (descriptive and exploratory) and spatial analysis, using geostatistics techniques (modeling of the spatial variability structure and inferences) and geoprocessing (map algebra). Except for phosphorus in 2005 and potassium in 2006, every other recommendation showed spatial dependence. Data analysis by the spatial method provided the identification of zones with deficient or excessive liming and fertilization which could not be defined by the conventional sampling method (zigzag).
\end{abstract}

KEYWORDS: geostatistics, geoprocessing, soil management.

\footnotetext{
${ }^{1}$ Extraído da Dissertação de Mestrado do primeiro autor.

${ }^{2}$ Eng $^{\mathrm{o}}$ Agrônomo, Doutorando em Energia na Agricultura, UNESP, Botucatu - SP, Fone: (0XX14) 3811.7119, rbatista@fca.unesp.br

${ }^{3}$ Eng ${ }^{\circ}$ Agric. Prof. Adjunto, Departamento de Engenharia Rural, UFES, Alegre - ES, limajss@yahoo.com.br

${ }^{4}$ Eng ${ }^{\mathrm{O}}$ Agrônomo, Prof. Adjunto, Departamento de Produção Vegetal, UFES, Alegre - ES.

${ }^{5}$ Graduando em Agronomia, Departamento de Engenharia Rural, UFES, Alegre - ES.

Recebido pelo Conselho Editorial em: 9-4-2007

Aprovado pelo Conselho Editorial em: 3-1-2008
} 


\section{INTRODUÇÃO}

A aplicação de fertilizantes e de corretivos a taxas variáveis apresenta-se como alternativa de grande potencial para minimizar os impactos negativos da atividade agrícola no ambiente. Apesar de essa prática já estar sendo utilizada comercialmente pelos produtores de soja, ainda faltam estudos para culturas perenes de grande valor econômico como o café.

Segundo MULLA et al. (1992), diferentemente da aplicação uniforme de fertilizantes e corretivos, que pode resultar em áreas com aplicações abaixo ou acima da dose necessária, a aplicação a taxas variáveis possibilita maior eficiência do uso de nutrientes com simultânea redução do potencial para poluição ambiental.

Visando ao melhor aproveitamento dos recursos agrícolas, muitos trabalhos sobre manejo localizado de culturas estão sendo desenvolvidos, especialmente em relação à aplicação de nutrientes no solo (WEIRICH NETO et al., 2006).

Segundo MANZATTO et al. (1999), a principal vantagem da agricultura de precisão é a possibilidade de aplicar insumos no local correto, no momento adequado, nas quantidades necessárias à produção agrícola, para áreas cada vez menores e mais homogêneas, tanto quanto a tecnologia e os custos envolvidos os permitam.

Nesse contexto, a geoestatística é importante ferramenta a ser considerada, uma vez que é capaz de propiciar o desenvolvimento de técnicas para a amostragem e descrever a variabilidade das características do meio físico de um sistema (LIBARDI et al., 1986). Ainda, segundo esses autores, os princípios básicos da experimentação, estabelecida por meio do método estatístico clássico, consideram que a variabilidade do solo ocorre de forma inteiramente aleatória e as coletas são feitas ao acaso, admitindo-se que seus atributos apresentem distribuição de freqüência normal. Entretanto, os atributos do solo apresentam intensa dependência espacial, necessitando, portanto, de análise geoestatística (VIEIRA, 2000).

O detalhamento da variabilidade espacial dos atributos da fertilidade do solo é possível conhecendo a posição geográfica dos pontos de amostragem. A partir da ordenação e do processamento desses dados, podem-se visualizar mapas temáticos em diferentes planos, utilizando técnicas de geoprocessamento e geoestatística (LAMPARELLI et al., 2001).

O manejo que se realiza no cultivo do café conilon, quanto à correção e à adubação do solo, é a aplicação com base nos valores médios de uma amostra composta, oriunda de subamostras coletadas em ziguezague na área, desconsiderando a variabilidade natural do solo. Em razão dessa variabilidade, é necessário estabelecer critério rigoroso de amostragem que permita, a partir de técnicas de amostragem, extrair informações representativas de uma determinada área. Segundo SILVA et al. (2007), a aplicação da agricultura de precisão na cultura do cafeeiro ainda não é uma realidade na agricultura brasileira, uma vez que existem poucos trabalhos sendo desenvolvidos nessa área.

O objetivo deste trabalho foi comparar a metodologia convencional de amostragem de solo com a análise espacial dos dados para a recomendação de calagem e de adubação de nitrogênio, fósforo e potássio em um solo sob cultivo do cafeeiro conilon.

\section{MATERIAL E MÉTODOS}

Os dados foram coletados durante as safras de 2004/05 e 2005/06 do café conilon, cultivado na Fazenda Experimental Bananal do Norte, pertencente ao Instituto Capixaba de Pesquisa, Assistência Técnica e Extensão Rural (INCAPER), localizada no município de Cachoeiro de Itapemirim, sul do Estado do Espírito Santo. A área experimental está compreendida nas coordenadas geográficas $20^{\circ} 45^{\prime} 17,31^{\prime \prime}$ sul e $41^{\circ} 17^{\prime} 8,86^{\prime}$ " oeste de Greenwich, com altitude média de $113 \mathrm{~m}$. 
A espécie de café utilizada foi a Coffea canephora Pierre, var. Robusta Tropical - 'Emcaper 8151 ', cultivada com espaçamento de $2,90 \times 0,9 \mathrm{~m}$.

As amostras de solo foram coletadas numa área situada no terço superior e médio de uma elevação com topo arredondado e vertente com perfil convexo, sendo o solo classificado como Latossolo Vermelho-Amarelo distrófico, com textura argilosa (EMBRAPA, 1999). Os valores da declividade ao longo da área variam de 0,06 a $33,52^{\circ}$, com média de $6,8^{\circ}$.

Dentro de uma área de 1,0 ha, foi construída uma grade irregular com 109 pontos amostrais, definidos como células. As coordenadas de cada ponto amostral foram adquiridas com auxílio de um GPS topográfico, modelo GTR-1, em cada uma das linhas de plantio e com espaçamento entre células de aproximadamente $10 \mathrm{~m}$ na linha (Figura 1).

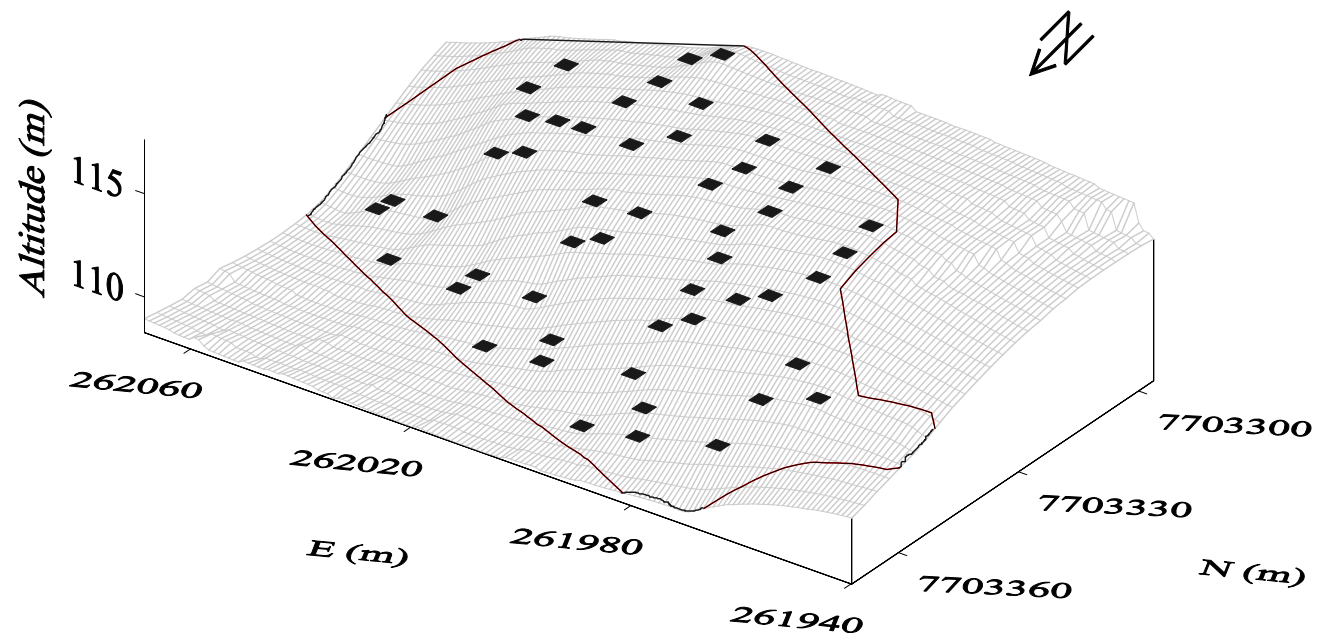

FIGURA 1. Representação digital do terreno com a distribuição espacial dos pontos amostrais. Digital representation of the land with spatial distribution of sample points.

Cada célula amostral foi composta de cinco plantas de café, compondo área de $13,05 \mathrm{~m}^{2}$. $\mathrm{O}$ georreferenciamento e a coleta de solos para cada ponto corresponderam ao centro de cada célula, conforme metodologia realizada por LEAL (2002), esquematizada na Figura 2.

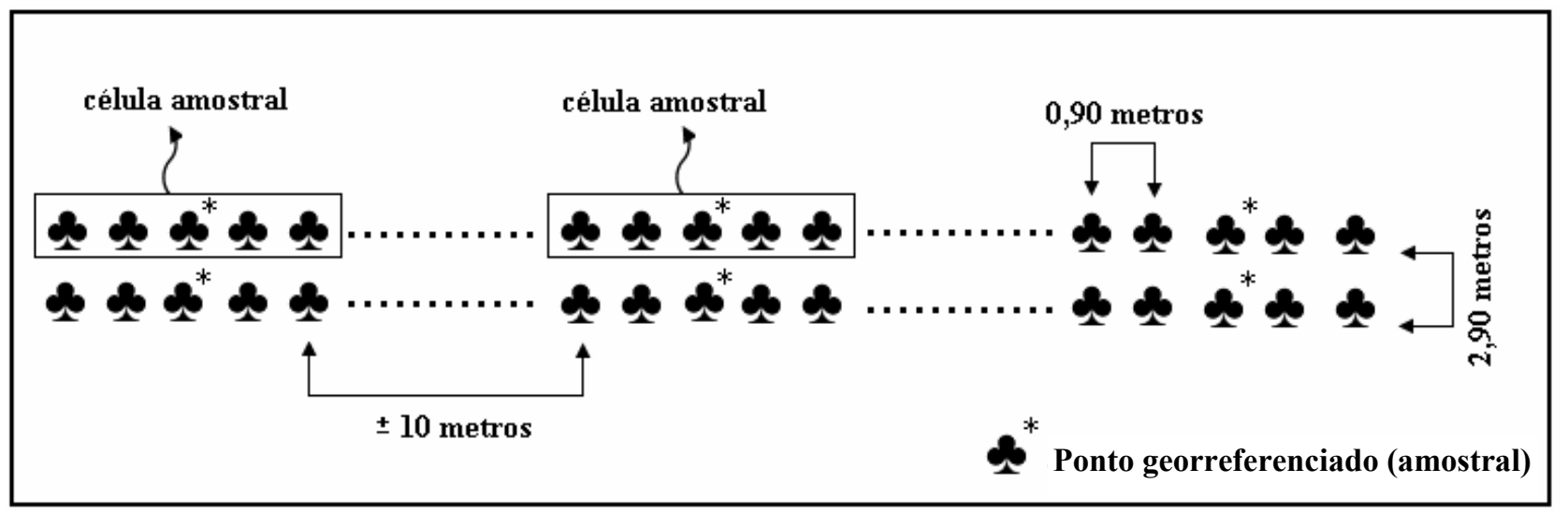

FIGURA 2. Esquema da metodologia de amostragem. Scheme of sampling methodology.

Para a caracterização química do solo, as amostras foram coletadas na projeção da copa do cafeeiro, na profundidade de 0-0,20 m, com auxílio de sonda inoxidável, para determinar os atributos: acidez ativa ( $\mathrm{pH}$ em água), acidez potencial $(\mathrm{H}+\mathrm{Al})$, cálcio trocável $\left(\mathrm{Ca}^{+2}\right)$, magnésio trocável $\left(\mathrm{Mg}^{2+}\right)$, acidez trocável $\left(\mathrm{Al}^{3+}\right)$, fósforo $(\mathrm{P})$ e potássio $\left(\mathrm{K}^{+}\right)$. Com base nas análises químicas, foram calculados os valores da soma de bases (SB), da capacidade de troca de cátions a $\mathrm{pH} 7$ (CTC), da percentagem de saturação por bases (V\%) e da percentagem de saturação por alumínio $(\mathrm{m} \%)$. 
Com os resultados dos pontos amostrais, para cada ano de avaliação, foram realizados os cálculos da determinação da dose de calcário $\left(\mathrm{t} \mathrm{ha}^{-1}\right)$ a ser recomendada para cada ponto amostrado pelo método da saturação por bases [eq.(1)], descrito por RIBEIRO et al. (1999), levando em consideração a cobertura total da área, a incorporação pela esparramação de $5 \mathrm{~cm}$ de profundidade e calcário com PRNT de 90\%.

$$
\mathrm{QC}=\mathrm{NC} \frac{\mathrm{SC}}{100} \frac{\mathrm{PF}}{20} \frac{100}{\mathrm{PRNT}}
$$

em que,

QC - quantidade de calcário a ser usado, $\mathrm{t} \mathrm{ha}^{-1}$;

$\mathrm{SC}$ - percentagem da superfície do terreno a ser coberta na calagem;

$\mathrm{PF}$ - profundidade que o calcário será incorporado, $\mathrm{cm}, \mathrm{e}$

PRNT - poder relativo de neutralização total do calcário a ser utilizado, \%.

Para a recomendação de adubação de $\mathrm{N}\left(\mathrm{kg} \mathrm{ha}^{-1}\right), \mathrm{P}\left(\mathrm{kg} \mathrm{ha}^{-1}\right) \mathrm{e} \mathrm{K}\left(\mathrm{kg} \mathrm{ha}^{-1}\right)$, foi realizado o cálculo de recomendação para cada ponto, em função do teor do elemento no solo e da produção esperada para o café conilon, conforme encontrado em FONSECA et al. (2004).

Inicialmente, os dados foram submetidos à análise estatística descritiva e exploratória, conforme proposto por LIBARDI et al. (1996) e GONÇALVES et al. (2001), para a verificação da presença de pontos discrepantes (outliers), ou seja, pontos que produzem alta variabilidade aos dados e alteram os valores de algumas medidas sensíveis a essas observações, como a média, a amplitude, o desvio-padrão e a assimetria da distribuição, contribuindo para a tendenciosidade dos dados, o que pode inviabilizar a aplicação das técnicas geoestatísticas. Na análise geoestatística, esses valores provocam forte impacto, principalmente na parte inicial do semivariograma. Esse fato pode conduzir a conclusões erradas sobre a variância do ruído (efeito pepita) e/ou existência de correlação espacial.

A geoestatística foi utilizada para verificar a autocorrelação espacial, investigar a magnitude da correlação entre as amostras e sua similaridade ou não com a distância. Para esta análise, foi utilizado o programa $\mathrm{GS}^{+}$(ROBERTSON, 2000). A dependência espacial foi analisada por meio de ajustes de semivariogramas, conforme VIEIRA (2000), com base nas pressuposições de estacionaridade da hipótese intrínseca, calculada pela eq.(2):

$$
\hat{\gamma}(\mathrm{h})=\frac{1}{2 \mathrm{~N}(\mathrm{~h})} \sum_{\mathrm{i}=1}^{\mathrm{N}(\mathrm{h})}\left[\left(\mathrm{x}_{\mathrm{i}}\right)-\mathrm{Z}\left(\mathrm{x}_{\mathrm{i}}+\mathrm{h}\right)\right]^{2}
$$

em que,

$\hat{\gamma}(h)$ - semivariância estimada;

$\mathrm{N}(\mathrm{h})$ - número de pares do atributo, e

$\mathrm{Z}\left(\mathrm{x}_{\mathrm{i}}\right), \mathrm{Z}\left(\mathrm{x}_{1}+\mathrm{h}\right)$ - valores dos atributos medidos na posição $\mathrm{x}_{\mathrm{i}}$ e $\mathrm{x}_{\mathrm{i}}+\mathrm{h}$, separados por um vetor h (distância entre amostras).

No ajuste de modelos teóricos (esférico, exponencial e gaussiano) aos valores calculados de $\hat{\gamma}(h)$, foram estimados os coeficientes: efeito pepita $\mathrm{C}_{0}$, patamar $\left(\mathrm{C}_{0}+\mathrm{C}_{1}\right)$, variância estrutural $\left(\mathrm{C}_{1}\right)$ e alcance de dependência espacial (a) para os semivariogramas. A escolha do melhor modelo baseou-se na menor soma do quadrado dos resíduos (SQR) e no coeficiente de determinação múltipla $\left(\mathrm{R}^{2}\right)$. Na seqüência, também foi utilizado o $\mathrm{R}^{2}$ da validação cruzada $\left(\mathrm{R}^{2}-\mathrm{VC}\right)$ (valores observados versus valores estimados). Para a análise do índice de dependência espacial (IDE) dos atributos, foi utilizada a relação definida no programa $\mathrm{GS}^{+}\left(\mathrm{C}_{1} / \mathrm{C}_{0}+\mathrm{C}_{1}\right)$ e os intervalos propostos por ZIMBACK (2001), que considera a dependência espacial fraca (IDE $\leq 25 \%)$; moderada $(25 \%<$ IDE $<75 \%$ ) e forte (IDE $\geq 75 \%$ ).

Com o ajuste dos modelos teóricos e a definição dos coeficientes para os semivariogramas, utilizou-se do método de estimativa de valores de atributos distribuídos no espaço a partir de 
valores adjacentes, conhecido como krigagem ordinária, que se trata de método de estimativa por médias móveis (LANDIM, 1998). A krigagem é o método de interpolação da geoestatística que usa a dependência espacial expressa no semivariograma entre pontos amostrais vizinhos para estimar valores em qualquer posição dentro do campo, sem tendência e com variância mínima (TRANGMAR et al., 1985).

Para a espacialização da recomendação localizada de calcário, foram utilizados os mapas confeccionados por krigagem ordinária para $\mathrm{Ca}^{+2}, \mathrm{Mg}^{+2}, \mathrm{~K}^{+}, \mathrm{H}+\mathrm{Al}$ e, em seguida, por meio de operações aritméticas sobre imagens, disponível no aplicativo do "software" Idrisi 32 (EASTMAN, 1999), foram calculados os valores da CTC, SB, V\% e, posteriormente, a determinação da quantidade de calcário na área com resolução de 1 x $1 \mathrm{~m}$.

Com a finalidade de comparar a superfície interpolada por krigagem com o método convencional de amostragem de solo, realizou-se, de acordo com a metodologia adotada no INCAPER, uma amostragem tradicional na camada de $0-0,20 \mathrm{~m}$ do solo, formada por 15 subamostras retiradas em caminhamento zigue-zague na lavoura, constituindo uma amostra composta para o cálculo da necessidade uniforme (pela média) de calagem e de adubação do solo, nos dois anos de avaliação (2005 e 2006).

Para a recomendação de fertilizantes, interpolaram-se pelo mesmo método os valores de $\mathrm{N}, \mathrm{P}$ e K no programa $\mathrm{GS}^{+}$e, em seguida, foram exportados para o Surfer 8.0 (GOLDEN SOFTWARE, 2002) para a confecção dos mapas.

Determinados os mapas de recomendações para calcário, N, P e K, construiu-se o mapa da diferença, por meio de subtração entre os valores interpolados, segundo a resolução adotada, e o valor médio do método convencional para cada um dos atributos. Em seguida, utilizou-se do módulo reclass no programa Idrisi 32 para reclassificar os mapas encontrados, de acordo com o valor médio obtido no método convencional de amostragem. Assim, foi possível obter duas zonas de necessidade, uma acima do valor médio (em excesso) e outra abaixo (em déficit). Na seqüência, calculou-se a percentagem de cada zona correspondente à área total.

\section{RESULTADOS E DISCUSSÃO}

Após os cálculos para recomendação espacial de calagem e de adubação para os dois anos de avaliação, realizaram-se a estatística descritiva e a distribuição de freqüência dos dados, conforme Tabela 1.

A recomendação de calagem no ano de 2005 (RC-05) apresentou distribuição de freqüência normal, a 5\% de probabilidade, pelo teste de Kolmogorov-Smirnov (KS). Apesar de os demais atributos não terem apresentado a normalidade, observa-se simetria na distribuição pela semelhança entre os valores de média e de mediana, e pelos valores de assimetria (Cs) e curtose (Ck) (Tabela 1).

De acordo com CRESSIE (1993), a normalidade dos dados não é uma exigência da geoestatística, desde que a distribuição dos dados não apresente extremidades da distribuição muito alongadas. Com exceção da recomendação de calagem para o ano de 2005 e 2006 (RC-05 e RC-06) e recomendação de adubação de fósforo (RP-05), as demais recomendações apresentam assimetria positiva. Segundo LIBARDI et al. (1996), na assimetria positiva, a mediana é menor que a média dos dados, mostrando, assim, a tendência para concentração de valores abaixo da média.

Com base no critério de WARRICK \& NIELSEN (1980), o coeficiente de variação apresentou-se baixo (C.V. $<12 \%$ ) para a recomendação de fósforo (RP-05) e de nitrogênio (RN06), nos anos de 2005 e 2006, respectivamente; médio $(12 \%<$ C.V. $<60 \%)$ para a recomendação de nitrogênio (RN-05), no ano de 2005, calcário (RC-06) e fósforo (RP-06), no ano de 2006, e potássio (RK-05 e RK-06), nos anos de 2005 e 2006; e apresentou-se alto (C.V. > 60\%) para a recomendação de calcário, no ano de 2005 (RC-05). 
TABELA 1. Estatística descritiva e distribuição de freqüência da recomendação de calcário (RC), nitrogênio (RN), fósforo (RP) e potássio (RK) para os anos de 2005 e 2006. Descriptive statistics and distribution of frequency of liming (LR), nitrogen NR), phosphorus PR) and potassium (KR) recommendation for 2005 and 2006.

\begin{tabular}{|c|c|c|c|c|c|c|c|c|c|}
\hline \multirow{2}{*}{ Recomendação } & \multirow{2}{*}{ Média } & \multirow{2}{*}{ Md } & \multirow{2}{*}{$\mathrm{S}$} & \multicolumn{2}{|c|}{ Valores } & \multirow{2}{*}{ C.V. } & \multirow{2}{*}{ Assimetria } & \multirow{2}{*}{ Curtose } & \multirow{2}{*}{$\begin{array}{c}\text { Teste } \\
\text { KS }\end{array}$} \\
\hline & & & & Mínimo & Máximo & & & & \\
\hline RC-05 (t ha $\left.{ }^{-1}\right)$ & 0,87 & 0,84 & 0,49 & 0,00 & 2,07 & 63,63 & $-0,15$ & $-0,14$ & $\mathrm{p}>0,20^{\mathrm{ns}}$ \\
\hline $\mathrm{RC}-06\left(\mathrm{t} \mathrm{ha}^{-1}\right)$ & 1,09 & 1,16 & 0,51 & 0,00 & 2,05 & 45,54 & $-1,37$ & 3,64 & $\mathrm{p}<0,15^{*}$ \\
\hline RN-05 (kg ha $\left.{ }^{-1}\right)$ & 342,05 & 320,00 & 58,37 & 280,00 & 500,00 & 17,06 & 1,19 & 1,00 & $\mathrm{p}<0,01^{*}$ \\
\hline RN-06 $\left(\mathrm{kg} \mathrm{ha}^{-1}\right)$ & 324,38 & 320,00 & 38,88 & 280,00 & 450,00 & 11,99 & 0,90 & 0,27 & $\mathrm{p}<0,01 *$ \\
\hline $\mathrm{RP}-05\left(\mathrm{~kg} \mathrm{ha}^{-1}\right)$ & 54,17 & 55,00 & 2,76 & 45,00 & 55,00 & 5,09 & $-3,07$ & 7,60 & $\mathrm{p}<0,01 *$ \\
\hline RP-06 $\left(\mathrm{kg} \mathrm{ha}^{-1}\right)$ & 47,50 & 47,50 & 10,20 & 30,00 & 65,00 & 21,47 & 0,09 & $-0,94$ & $\mathrm{p}<0,05^{*}$ \\
\hline RK-05 $\left(\mathrm{kg} \mathrm{ha}^{-1}\right)$ & 121,83 & 100,00 & 32,58 & 100,00 & 170,00 & 26,75 & 0,82 & $-1,34$ & $\mathrm{p}<0,01 *$ \\
\hline RK-06 $\left(\mathrm{kg} \mathrm{ha}^{-1}\right)$ & 115,39 & 105,00 & 33,96 & 80,00 & 210,00 & 29,43 & 0,81 & $-0,22$ & $\mathrm{p}<0,01^{*}$ \\
\hline
\end{tabular}

Md - mediana; s - desvio-padrão; C.V. - coeficiente de variação; ${ }^{\text {ns }}$ - distribuição normal, pelo teste de KolmogorovSmirnov (KS), a 5\% de probabilidade; * Distribuição não-normal.

Os resultados da análise geoestatística (Tabela 2 e Figura 3), com exceção da recomendação de P para 2005 (RP-05) e K para 2006 (RK-06), mostraram que todos os atributos analisados apresentaram estrutura de dependência espacial na escala de amostragem adotada. As recomendações RC-05, RC-06 e RK-05 ajustaram-se ao modelo exponencial, e RN-05 e RN-06 ajustaram-se ao modelo esférico com $\mathrm{R}^{2}$ entre $73 \%$ e 94\%. MARQUES JÚNIOR et al. (2000) ajustaram o modelo esférico estudando a variabilidade espacial de atributos químicos do solo, na cultura do café. Os atributos que apresentaram ausência de dependência espacial (RP-05 e RK-06), ou seja, efeito pepita puro, revelam que as amostras não foram coletadas em intervalos suficientemente pequenos para mostrar o comportamento espacial desses atributos. Do ponto de vista prático, isso está relacionado com as doses de adubos fosfatados e potássios utilizadas para a manutenção da cultura, forma de aplicação no solo e metodologia para o cálculo de recomendação.

Os valores dos alcances encontrados nos semivariogramas têm importância na determinação do limite da dependência espacial, podendo ser também indicativo do intervalo entre unidades de mapeamento de solos (TRANGMAR et al., 1985). Na Figura 3, observa-se que, em 2005, a RP-05 apresentou ausência de dependência espacial e, no ano seguinte, a RP-06 apresentou correlação entre as amostras até o alcance de 12,0 m. Esse alcance está relacionado com a baixa mobilidade do P no solo. Entretanto, em 2005, a RK-05 apresentou alcance de 11,1 m e, em 2006, ausência de dependência espacial, fato esse relacionado com a grande mobilidade desse atributo no solo. Segundo SILVA et al. (2007), a prática de manejo adotado no cultivo do café contribui para a redução do alcance, ou seja, mais rapidamente obter a independência entre amostras.

TABELA 2. Modelos e parâmetros estimados dos semivariogramas para as recomendações de calcário (RC), nitrogênio (RN), fósforo (RP) e potássio (RK) para os anos de 2005 e 2006. Semivariogram estimated models and parameters for liming (LR), nitrogen (NR), phosphorus (PR) and potassium (KR) recommendations for 2005 and 2006.

\begin{tabular}{llrrrrrrr}
\hline \multicolumn{1}{c}{ Recomendação } & \multicolumn{1}{c}{ Modelo } & $\begin{array}{r}\mathrm{a} \\
(\mathrm{m})\end{array}$ & $\mathrm{C}_{0}$ & $\mathrm{C}_{0}+\mathrm{C}_{1}$ & IDE $(\%)$ & $\begin{array}{r}\mathrm{R}^{2} \\
(\%)\end{array}$ & $\begin{array}{r}\mathrm{R}^{2}-\mathrm{CV} \\
(\%)\end{array}$ & Signif. \\
\hline $\mathrm{RC}-05\left(\mathrm{t} \mathrm{ha}^{-1}\right)$ & Exponencial & 39,00 & 0,51 & 1,04 & 51,00 & 84,00 & 15,50 & 0,0000 \\
$\mathrm{RC}-06\left(\mathrm{t} \mathrm{ha}^{-1}\right)$ & Exponencial & 19,00 & 0,24 & 0,91 & 74,00 & 73,00 & 13,30 & 0,0000 \\
$\mathrm{RN}-05\left(\mathrm{~kg} \mathrm{ha}^{-1}\right)$ & Esférico & 44,10 & 0,39 & 1,09 & 64,20 & 94,00 & 42,80 & 0,0000 \\
$\mathrm{RN}-06\left(\mathrm{~kg} \mathrm{ha}^{-1}\right)$ & Esférico & 30,00 & 0,29 & 1,09 & 74,00 & 91,00 & 46,00 & 0,0000 \\
$\mathrm{RP}-05\left(\mathrm{~kg} \mathrm{ha}^{-1}\right)$ & EPP & 0,00 & 1,00 & 1,00 & 0,00 & 0,00 & 0,00 & 0,0795 \\
$\mathrm{RP}-06\left(\mathrm{~kg} \mathrm{ha}^{-1}\right)$ & Esférico & 12,00 & 0,24 & 1,04 & 77,00 & 90,00 & 11,50 & 0,0000 \\
$\mathrm{RK}-05\left(\mathrm{~kg} \mathrm{ha}^{-1}\right)$ & Exponencial & 11,10 & 0,29 & 1,00 & 71,00 & 94,00 & 8,41 & 0,0391 \\
RK-06 $\left(\mathrm{kg} \mathrm{ha}^{-1}\right)$ & EPP & 0,00 & 1,00 & 1,00 & 0,00 & 0,00 & 0,00 & 0,0150 \\
\hline
\end{tabular}

EPP - efeito pepita puro; $C_{0}$ - efeito pepita; $C_{0}+C_{1}$ - patamar; IDE - índice de dependência espacial $\left(C_{1} / C_{0}+C_{1}\right)$; a alcance; $\mathrm{R}^{2}$ - coeficiente de determinação do modelo; $\mathrm{R}^{2}$ - VC - coeficiente de determinação da validação cruzada, Signif. - nível de significância da validação cruzada. 

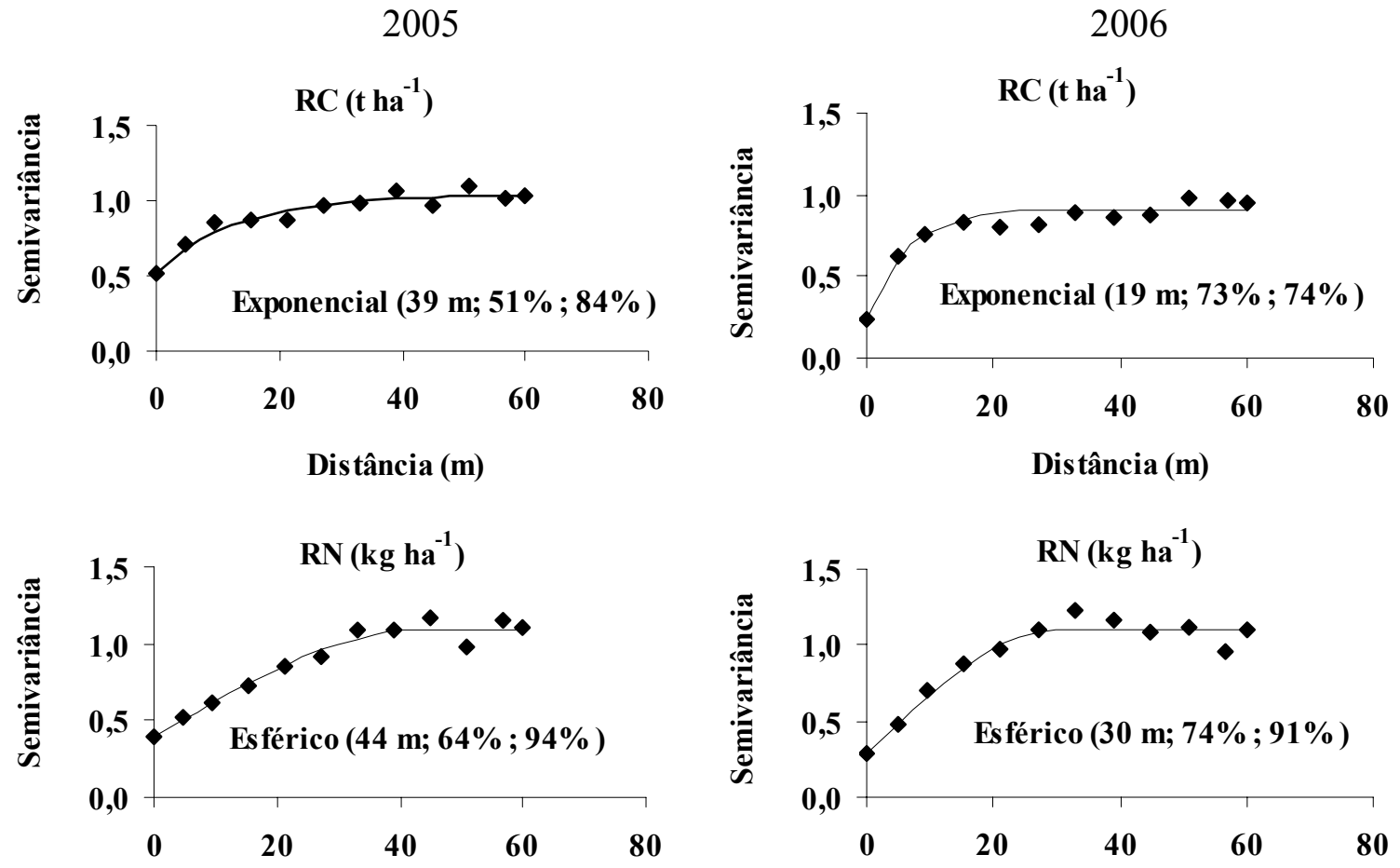

Distância (m)

Distância (m)
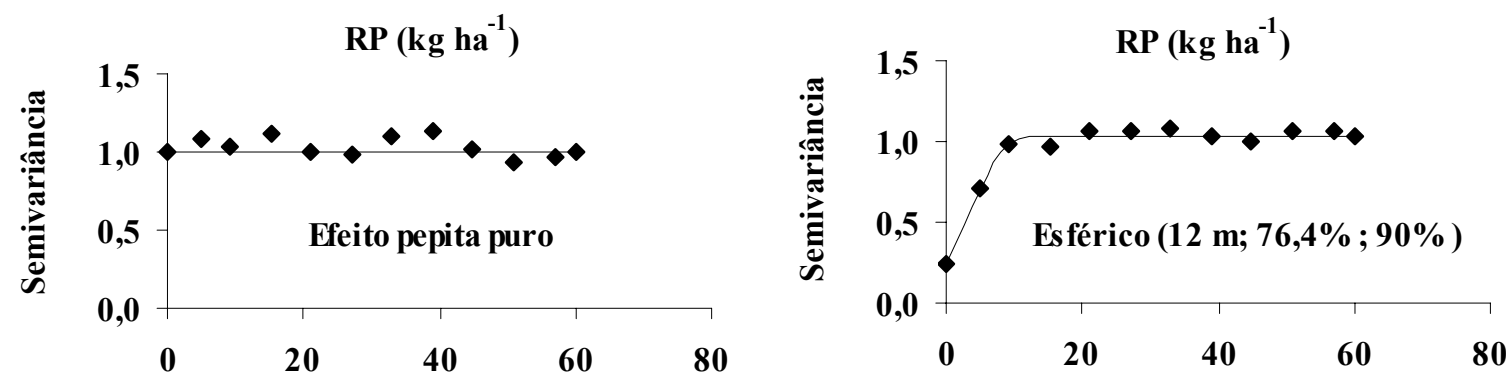

Distância (m)

Distância (m)
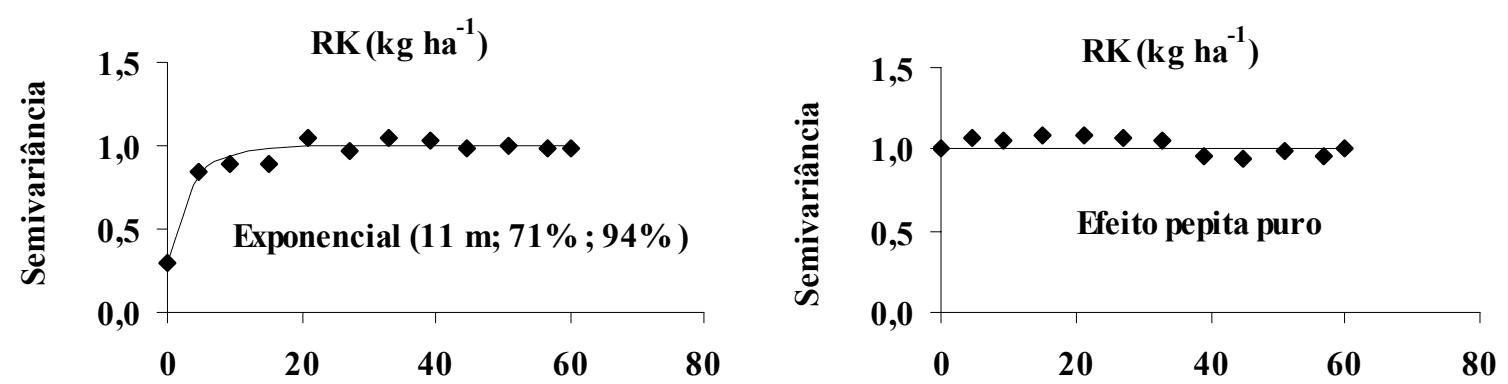

Distância (m)

Distância (m)

FIGURA 3. Modelos e parâmetros (a, IDE e $\mathrm{R}^{2}$, respectivamente) dos semivariogramas para recomendação de calcário (RC), nitrogênio (RN), fósforo $(\mathrm{RP})$ e potássio $(\mathrm{RK})$, em 2005 e 2006. Semivariogram models and parameters (a, IDE and $\mathbf{R}^{\mathbf{2}}$, respectively) for liming (LR), nitrogen (NR), phosphorus (PR) and potassium (KR) recommendation in 2005 and 2006.

Quanto ao grau de dependência espacial (IDE), apenas RP-06 apresenta forte dependência espacial, enquanto os demais atributos apresentam moderada dependência espacial, com exceção das RP-05 e RK-06, que proporcionam efeito pepita puro, segundo classificação de ZIMBACK (2001). 
Na Figura 4, são apresentados os mapas temáticos para a recomendação de calagem (RC) e de adubação com N, P e K, construídos por krigagem ordinária.

Observa-se que os mapas de RP-06 e de RK-05 indicam alta variabilidade das classes de aplicação de doses de insumos, ou seja, descontinuidade da dependência espacial em função dos menores alcances obtidos.

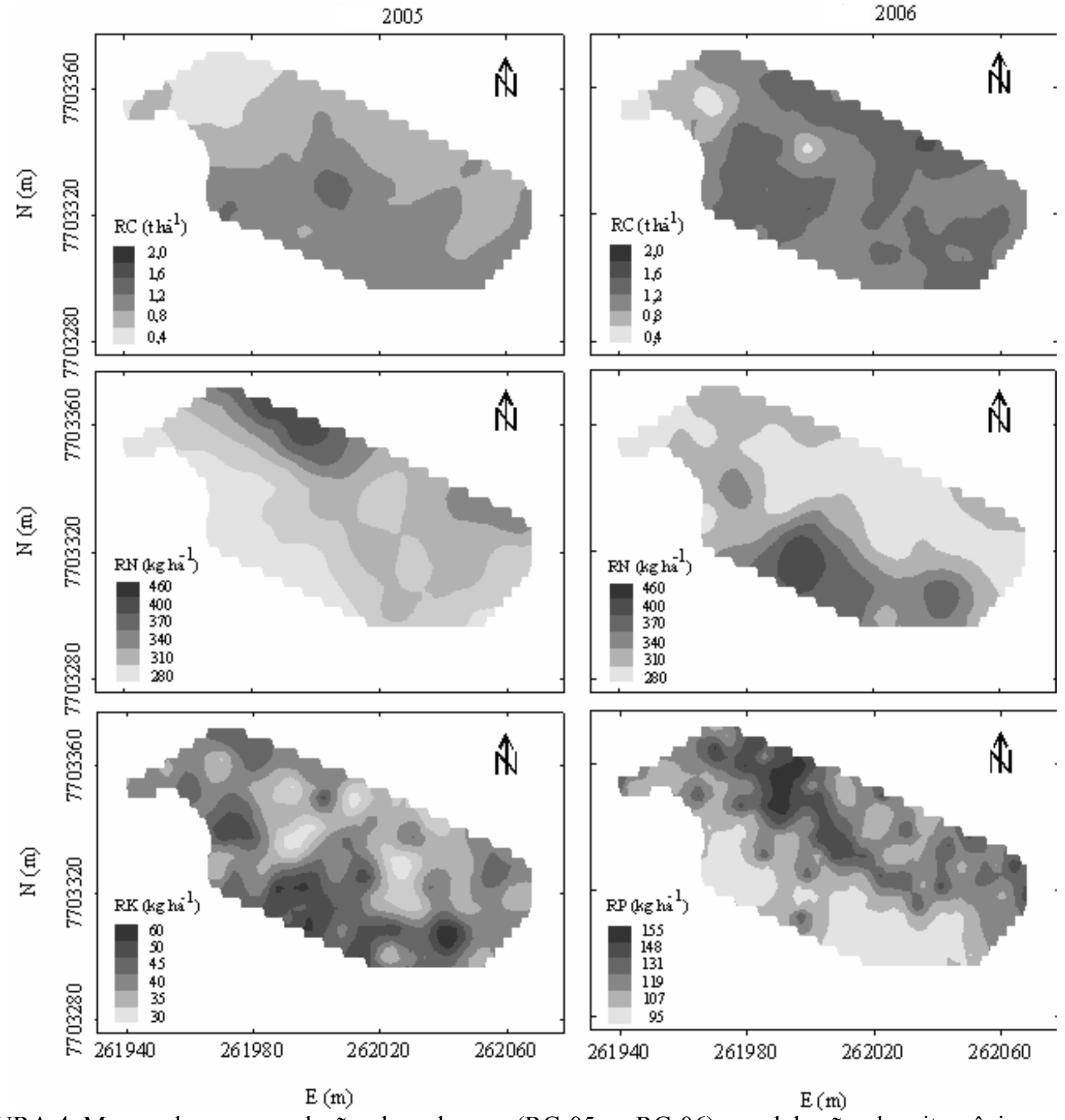

FIGURA 4. Mapas de recomendação de calagem (RC-05 e RC-06) e adubação de nitrogênio (RN-05 e RN-06), fósforo (RP-06) e potássio (RK-05) para 2005 e 2006. Maps of liming recommendation (LR-05) and (LR-06) and nitrogen (NR-05 and NR-06), phosphorus (PR-06) and potassium (KR-05) fertilization for 2005 and 2006.

Na Figura 5, são apresentados os mapas da diferença entre os dois métodos de recomendação de calcário e adubação com $\mathrm{N}, \mathrm{P}\left(\mathrm{P}_{2} \mathrm{O}_{5}\right)$ e $\mathrm{K}\left(\mathrm{K}_{2} \mathrm{O}\right)$. Nesses mapas, a cor escura corresponde às áreas em excesso, e a cor clara corresponde às áreas em déficit, tendo como padrão os valores médios na legenda calculados a partir do método convencional, bem como entre parênteses a percentagem da área em excesso e em déficit; portanto, os mapas indicam as regiões que estão abaixo e acima da recomendação pelo método convencional. 
2005

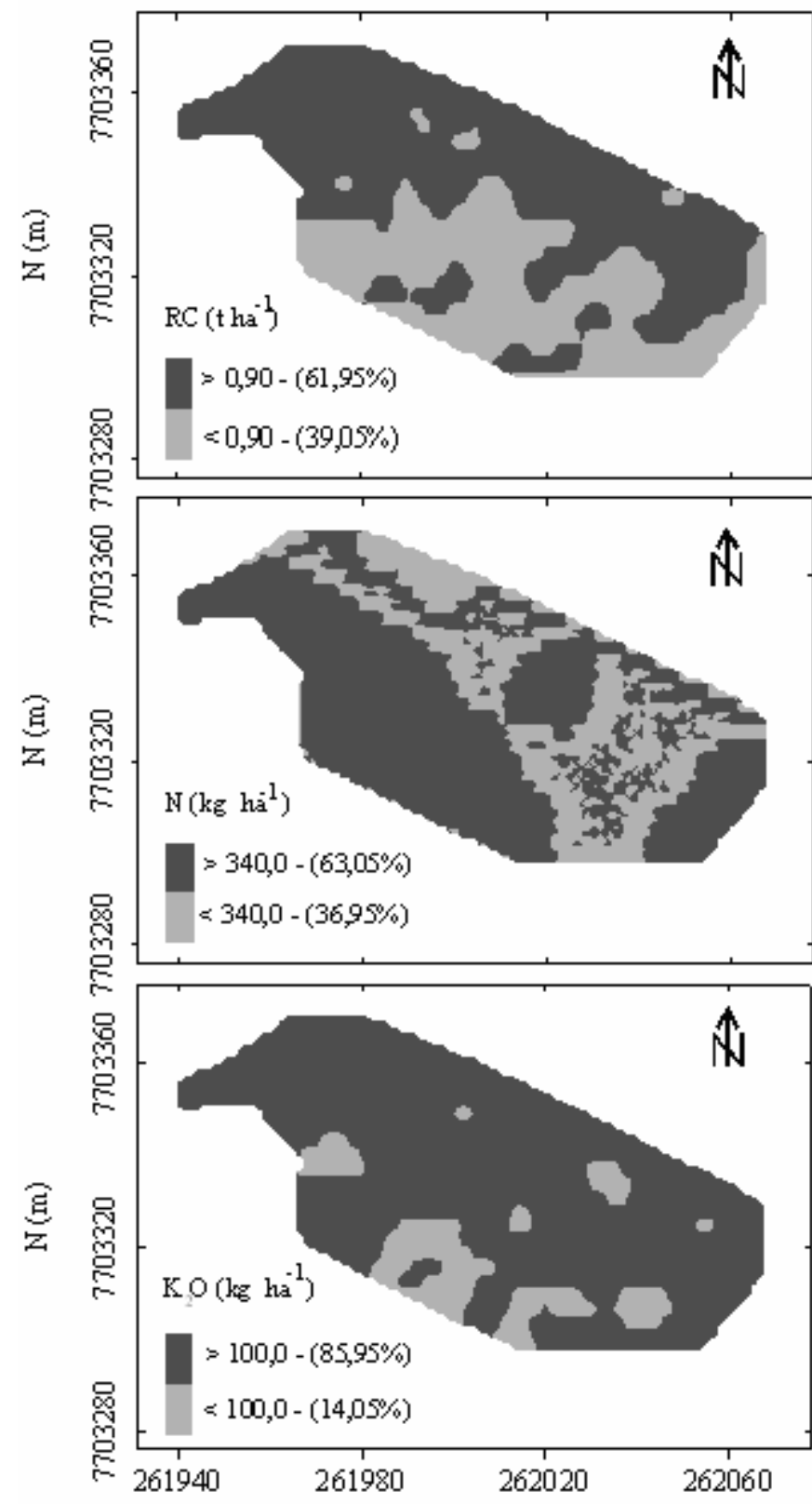

2006
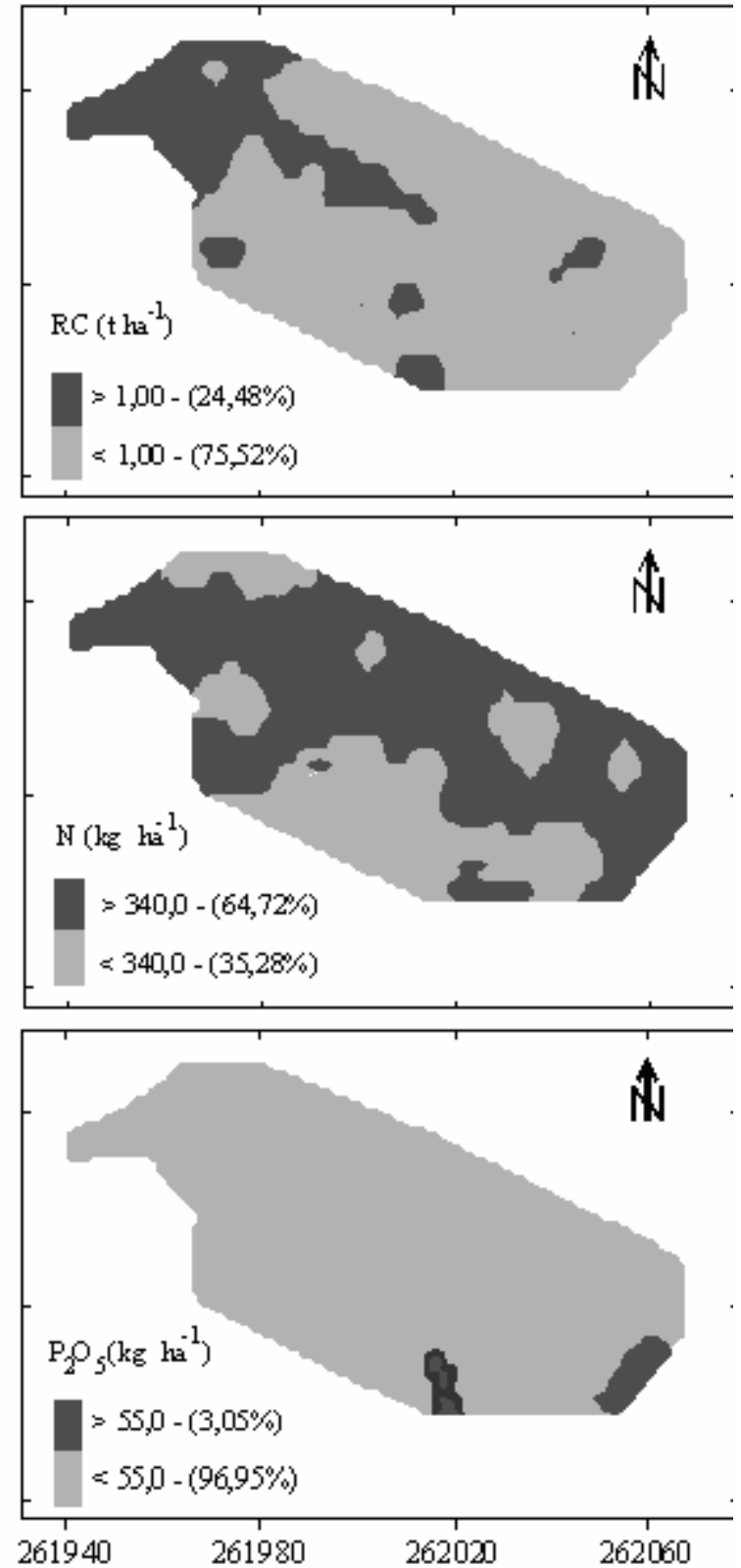

$E(m)$

$E(m)$

FIGURA 5. Mapas da diferença entre a recomendação pelo método convencional e a análise espacial do calcário $(\mathrm{RC})$ e adubação de nitrogênio $(\mathrm{N})$, fósforo $\left(\mathrm{P}_{2} \mathrm{O}_{5}\right)$ e potássio $\left(\mathrm{K}_{2} \mathrm{O}\right)$ para 2005 e 2006. Maps of the difference between the recommendation by the conventional method and the liming spatial analysis (LR) and the fertilization of nitrogen $(\mathrm{N})$, phosphorus $\left(\mathrm{P}_{2} \mathrm{O}_{5}\right)$ and potassium $\left(\mathrm{K}_{2} \mathrm{O}\right)$ for 2005 and 2006.

A recomendação calculada pelo método convencional de amostragem do solo, que foi usada como padrão, indicou 0,9 e $1,0 \mathrm{t} \mathrm{ha}^{-1}$ de calcário, $340 \mathrm{~kg} \mathrm{ha}^{-1} \mathrm{de} \mathrm{N}, 55 \mathrm{~kg} \mathrm{ha}^{-1}$ de $\mathrm{P}_{2} 0_{5}$, e 100 e $158 \mathrm{~kg} \mathrm{ha}^{-1}$ de $\mathrm{K}_{2} \mathrm{O}$ para os anos de 2005 e 2006 , respectivamente.

Analisando os resultados obtidos pelos dois métodos, observam-se áreas com aplicações acima do padrão (excesso) em mais de 50\% da área para a RC-05, RN-05, RN-06 e RK-05, e aplicações abaixo (déficit) para RC-06 e RP-06. Comparando a recomendação pela amostragem convencional (ziguezague) com a análise espacial, verifica-se que a aplicação de $\mathrm{P}_{2} 0_{5}$ (2006), pela média $\left(55 \mathrm{~kg} \mathrm{ha}^{-1}\right)$, proporciona percentagem de, aproximadamente, $97 \%$ de déficit, não atendendo às exigências agronômicas da cultura, o que pode influenciar no seu rendimento. No caso do $\mathrm{K}_{2} \mathrm{O}$ 
(2005), a dose uniforme de $158 \mathrm{~kg} \mathrm{ha}^{-1}$ proporcionaria excesso desse nutriente no solo em $86 \%$, levando a custos desnecessários e superadubação.

YANG et al. (1999) encontraram aumentos da produção e reduções no uso de fertilizantes associados à prática das taxas variáveis de aplicação de $\mathrm{N}, \mathrm{P}, \mathrm{K}$ para sorgo irrigado. Portanto, a opção de adoção do manejo uniforme ou variar as taxas de aplicação na área, de acordo com as diferenças da fertilidade, contribui para reduzir o total de calcário, $\mathrm{N}$ e $\mathrm{K}$ aplicados, as perdas e problemas por aplicações excessivas, além de diminuir o custo de produção.

Para o mapa de recomendação de calcário, em 2005 (RC-05), nota-se que 61,95\% da área estão acima (excesso) com a aplicação da dose única recomendada de $0,90 \mathrm{t} \mathrm{ha}^{-1}$. Esse excesso de calcário pode levar a diversos problemas bem conhecidos de natureza química, como a diminuição da disponibilidade de micronutrientes no solo e, conseqüentemente, desequilíbrio nutricional nas plantas. Em 2006, verifica-se que 75,52\% da área apresentam déficit da dose única recomendada $\left(1,0 \mathrm{t} \mathrm{ha}^{-1}\right)$. Nessa análise comparativa, verifica-se que a aplicação de corretivos e fertilizantes utilizando taxas variáveis, em regiões específicas no solo, possibilita minimizar os custos de produção e eficiência do uso de insumos.

\section{CONCLUSÕES}

Com exceção da recomendação de fósforo no ano de 2005 e potássio em 2006, todas as demais recomendações apresentaram dependência espacial.

Os atributos fósforo, em 2006, e potássio, em 2005, apresentaram os menores alcances, evidenciando a baixa continuidade no solo.

A análise dos dados pelo método espacial possibilitou identificar zonas de déficit ou excesso de calagem e de adubação na área, que não poderiam ser definidas com o método convencional de amostragem (ziguezague).

\section{AGRADECIMENTOS}

Ao CNPq, pela bolsa concedida ao primeiro autor, e ao INCAPER, pela disponibilidade da área.

\section{REFERÊNCIAS}

CRESSIE, N.A. Statistics for spatial data. New York: John Wiley, 1993. 900 p.

EASTMAN, J.R. IDRISI32 for Windows: User's guide. Version 32.11. Software de sistema de informação geográfica (software). Worcester: Clark University, 1998. 192 p.

EMBRAPA. EMPRESA BRASILEIRA DE PESQUISA AGROPECUÁRIA. Centro Nacional de Pesquisa de Solos. Sistema brasileiro de classificação de solos. Rio de Janeiro, 1999. 412 p.

FONSECA, A.F.A.; FERRÃO, M.A.G.; FERRÃO, R.G.; VERDIN FILHO, A.C.; VOLPI, P.C.; MARQUES, E.M.G. Incaper 8142: Conilon Vitória: variedade clonal melhorada de café conilon. Vitória: INCAPER, 2004. (Documentos 127).

GONÇALVES, A.C.A; FOLEGATTI, M.V.; da MATA, J.D.V. Análises exploratórias e geoestatística da variabilidade de propriedades físicas de um Argissolo Vermelho. Acta Scientiarum, Maringá, v.23, n.5, p.1149-57, 2001.

LAMPARELLI, R.A.C.; ROCHA, J.V.; BHORGUI, E. Geoprocessamento e agricultura de precisão - Fundamentos e Aplicações. Guaíba: Agropecuária, 2001. v.1. 118 p.

LANDIM, P.M.B. Análise estatística de dados geológicos. São Paulo: UNESP, 1998. 226 p. 
LEAL, J.C.G. Mapeamento da produtividade na colheita mecanizada do café. $2002.74 \mathrm{f}$. Dissertação (Mestrado em Máquinas Agrícolas) - Escola Superior de Agricultura "Luiz de Queiroz”, Universidade de São Paulo, Piracicaba, 2002.

LIBARDI, P.L.; MANFRON, P.A.; MORAES, S.O.; TUON, R.L. Variabilidade da umidade gravimétrica de um solo hidromórfico. Revista Brasileira de Ciências do Solo, Viçosa, v.20, n.1, p.1-12, 1996.

LIBARDI, P.L.; PREVEDELLO, C.L.; PAULETTO, E.A.; MORAES, S.O. Variabilidade espacial da umidade, textura e densidade de partículas ao longo de uma transeção. Revista Brasileira de Ciências do Solo, Viçosa, v.10, n.2, p.85-90, 1986.

MANZATTO, C.V.; BHERING, S.B.; SIMÕES, M. Agricultura de precisão: propostas e ações da Embrapa Solo. EMBRAPA Solos, 1999. Disponível em: http://www.cnps.embrapa.br. Acesso em: 15 jun. 2006.

MARQUES JÚNIOR, J.; SANCHEZ, R.B.; PEREIRA, G.T. Variabilidade espacial de propriedades químicas e físicas de latossolos em diferentes superfícies geomórficas sob cultivo de café, em Patrocínio - MG. In: Balastreire, L.A. (Org.). O Estado da arte da agricultura de precisão. Piracicaba: ESALQ/USP, 2000. p.105-12.

MULLA, D.J.; BHATTI, A.U.; HAMMOND, M.W.; BENSON, J.A.A. A comparison of winter wheat yield and quality under uniform versus spatially variable fertilizer management. Agriculture, Ecosystems and Environment, Amsterdam, v.8, n.3, p.301-11, 1992.

OLDEN SOFTWARE. Surfer version 8.00 - Feb 11 2002. Surface mapping system. Colorado: Golden Software, 1993-2002. 1 CD-ROM.

RIBEIRO, A.C.; GUIMARÃES, P.T.G.; ALVAREZ, V.V.H. (Eds.). Recomendação para o uso de corretivos e fertilizantes em Minas Gerais. Viçosa: CFSEMG, 1999. 359 p.

ROBERTSON, G.P. GS ${ }^{+}$: Geostatistics for the environmental sciences - GS ${ }^{+}$User's Guide. Plainwell: Gamma Desing Software, 2000. 152 p.

SILVA, F.M.; SOUZA, Z.M.; FIGUEIREDO, C.A.P.; MARQUES JÚNIOR, J.; MACHADO, R.V. Variabilidade espacial de atributos químicos e de produtividade na cultura do café. Ciência Rural, Santa Maria, v.37, n.2, p.401-7, 2007.

TRANGMAR, B.B.; YOST, R.S.; UEHARA, G. Applications of geostatistics to spatial studies of soil properties. Advances in Agronomy, San Diego, v.38, n.1, p.45-94, 1985.

VIEIRA, S.R. Geoestatística em estudos de variabilidade espacial do solo. In: NOVAIS, R.F.; ALVARE, V.V.H.; SCHAEFER, C.E.G.R. (Eds.). Tópicos em ciência do solo. Viçosa: Sociedade Brasileira de Ciência do Solo, 2000. v.1, p.1-54.

WARRICK, A.W.; NIELSEN, D.R. Spatial variability of soil physical properties in the field. In: HILLEL, D. (Ed.). Applications of soil physics. New York: Academic, 1980. p.319-44.

WEIRICH NETO, P.H.; SVERZUT, C.B.; SCHIMANDEIRO, A. Necessidade de fertilizante e calcário em área sob sistema plantio direto considerando variabilidade espacial. Revista brasileira de engenharia agrícola e ambiental, Campina Grande, v.10, n.2, p.338-43, 2006.

YANG, C.; ANDERSON, G.L.; KING JR., J.H.; CHANDLER, E.K. Comparison of uniform and variable rate fertilization strategies using grid soil sampling, variable rate technology, and yield monitoring. In: INTERNATIONAL CONFERENCE ON PRECISION AGRICULTURE, 4., 1998. Minneapolis. Proceedings... Madison: ASA/ CSSA/ SSSA, 1999. p. 675-86.

ZIMBACK, C.R.L. Análise espacial de atributos químicos de solos para fins de mapeamento da fertilidade. 2001. 114 f. Tese (Livre-Docência em Levantamento do Solo e Fotopedologia) Faculdade de Ciências Agronômicas, Universidade Estadual Paulista, Botucatu, 2001. 